\title{
Biogas y compost a partir de residuos
}

\section{Biogas and compost from waste}

Presentación: 6 y 7 de octubre 2020.

\section{Doctorando:}

\section{Ivone Elisabet Carroza}

Grupo de Ingeniería Aplicada a los Bioprocesos, Facultad Regional Neuquén, Universidad Tecnológica Nacional - Argentina. ivynqn@gmail.com

\section{Director:}

\section{José Luis Boiardi}

\section{Co-director:}

\section{Marcela Noemí Gatti}

\section{Resumen}

El incremento de residuos asociados al crecimiento de la población es un problema grave en nuestra sociedad. La posibilidad de biometanización es una alternativa amigable con el medio ambiente ya que contribuye al procesamiento de centenares de toneladas de basura que se acumulan en nuestras ciudades. En Neuquén, la fracción orgánica de los residuos alcanza el $44 \%$ y es una potencial fuente de energía. Este puede ser valorizado por medio de biogas y compost.

El potencial metanogénico se puede medir empleando el método de la jeringa que tiene la ventaja de ser un procedimiento experimental de bajo costo. El presente trabajo consistió en comparar la cantidad de biogas generada en función de la concentración de sólidos totales. El potencial de biogas es el volumen de producto generado por la cantidad de sustrato, en este caso, la fracción orgánica de los residuos. Se realizó el ensayo usandolo como sustrato y empleando el agua residual de laguna anaerobia como inóculo. Se utilizó jeringas de $60 \mathrm{ml}$ a $35^{\circ} \mathrm{C}$ con concentraciones sustrato $5 \%, 10 \%, 15 \%, 20 \%$ y $40 \%$ de sólidos totales de la fracción orgánica y $10 \mathrm{ml}$ de inóculo de agua residual de laguna anaerobia.

Se determinó que la condición más favorable fue para las concentraciones de 5 \% y 20 \% de sólidos totales.

Además, se evaluó la preparación de un acelerante para el digestato por el método de zucconi.

Palabras claves: FORSU, fracción orgánica de los residuos, potencial de biogas, compost, digestato.

\begin{abstract}
The increase in waste associated with population growth is a serious problem in our society. The possibility of biomethanization is an environmentally friendly alternative as it contributes to the processing of hundreds of tons of waste that accumulate in our cities. In Neuquén, the organic fraction of waste reaches $44 \%$ and is a potential source of energy. This can be recovered by means of biogas and compost.

The methanogenic potential can be measured using the syringe method which has the advantage of being a lowcost experimental procedure. The present work consisted of comparing the amount of biogas generated as a function of the concentration of total solids. The biogas potential is the volume of product generated by the amount of substrate, in this case, the organic fraction of the waste. The test was carried out using it as a substrate and using the anaerobic lagoon wastewater as inoculum. Syringes of $60 \mathrm{ml}$ at $35^{\circ} \mathrm{C}$ were used with substrate concentrations of $5 \%, 10 \%, 15$ $\%, 20 \%$ and $40 \%$ of total solids of the organic fraction and $10 \mathrm{ml}$ of anaerobic lagoon wastewater inoculum.

It was determined that the most favourable condition was for concentrations of $5 \%$ and $20 \%$ total solids. In addition, the preparation of an accelerator for the digestate was evaluated by the zucconi method.
\end{abstract}

Keywords: FORSU, organic waste fraction, biogas potential, compost, digestate. 
El problema de la basura es un tema de interés mundial (Rattanapan et al., 2019) y es una consecuencia inevitable de las actividades humanas (Van et al., 2020). La generación creciente de los residuos presenta serias dificultades (Westerholm et al., 2020). Estos desechos generan organismos patógenos, vectores, enfermedades en personas y contaminación ambiental. (Barragan et al., 2010) Cuando se arrojan los desechos de comida directamente al vertido estos producen afectaciones negativas en el medio ambiente (Toledo et al., 2019). En cambio, si se emplea la digestión anaerobia para tratar la fracción orgánica de los residuos producen biogas y digestato, que adquieren valor. El digestato puede usarse como enmienda por sus propiedades fertilizantes. (Gallardo-Izquierdo, et. al 2013) y tiene la ventaja de mejorar las reservas de suelo, absorción de nutrientes y evitar liberación de $\mathrm{NO}_{2}$ (Azim et al., 2018)

Aunque el proceso de digestion anaerobia y compostaje son tecnología antigüas, existen perspectivas de conocimiento que merecen investigación y desarrollo (Pramanik et al., 2019). Por ejemplo, los problemas asociados a la digestion anaerobica de aguas residuales (AR) junto con la fracción orgánica de los residuos (FORSU) y la posibilidad de optimización este proceso (Pramanik et al., 2019), y asimismo, el uso de digestato como mejora de las reservas de suelo y absorción de nutrientes (Azim et al., 2018).

La información de la composición de los residuos es fundamental para la gestión y tratamiento de los mismos.(Villalba et al., 2020). En la Provincia del Neuquén hay 550.344 habitantes que producen un total $455 \mathrm{Tn} / \mathrm{d}$. (Deluca y Giorgi, 2015). En la ciudad de Neuquén, año 2015, habitaban 245.419 personas (Neuquén, 2015) y la producción de RSU era de 209 Tn/d. Además, en esta ciudad, el 44 \% corresponde a la FORSU. Definiendo la fracción orgánica de los residuos (FORSU) aquella constituida principalmente por restos de comida elaborada y desecho orgánicos como: verduras, frutas y desechos de infusions. Es decir, la FORSU tiene características heterogéneas.

La producción de biogás de los residuos, consiste en determinar el biogas generado cuando es agregado inóculo al sustrato o residuos. (Holliger et al., 2016). Este inóculo está activo en condiciones operacionales definidas para medir la producción de metano. El presente estudio tiene por objetivo establecer la cantidad de biogás a partir de FORSU de la ciudad de Neuquén usando como inóculo agua residual de laguna anaerobia ARLA. A través del método experimental de jeringa denominado el test de Hohemheim (HBT).

Además se preparó un inóculo para el digestato a partir de los mismos residuos, dado que se deben emplear microorganismos eficaces (Gallardo-Izquierdo, et. al , 2013). La calidad de éste puede medirse por el test de fitotoxicidad de Zucconi.

\section{Desarrollo}

\subsection{Toma de muestra y caracterización de residuos}

En Argentina la norma de referencia para la clasificación de residuos domésticos es ASTM D5231(Villalba et al., 2020). El método describe cómo medir la composición de los residuos sólidos urbanos sin procesar usando clasificación manual. La muestra de FORSU recolectada se reduce en tamaño. Luego se seca a $60^{\circ} \mathrm{C}$ para conservar sus propiedades y se reserva para su uso. El secado es una etapa previa para poder triturar y homogeneizar los residuos.(Carneiro, 2005). Se conservan a $4{ }^{\circ} \mathrm{C}$ en heladera. Los residuos secos se preparan con agua destilada y son procesados para homogeneizarlos.

La muestra de agua residual de laguna anaerobia (ARLA) se tomó de la planta de tratamiento de efluentes, EPAS, ubicada en Plaza Huincul, ciudad de Neuquén. Además, se recolectó la FORSU separada de origen correspondiente a familias de 4 integrantes, para preparar inóculo y comenzar el tratamiento y valorización digestato.

Se determinó en las muestras sólidos totales, sólidos volátiles, $\mathrm{pH}$, conductividad y DQO, bajo normas estandarizadas; los métodos usados fueron bajo las normas estandarizadas para aguas (APHA, 1989) y las normas bolivianas para residuos sólidos urbanos 742-760 (MDH, 1996). El pH se determinó con un pHmetro marca Hanna modelo HI 8424, previamente calibrado con buffer $\mathrm{pH}=4$ y $\mathrm{pH}=7$. La conductividad se midió con un conductímetro portátil calibrado marca Aquacombo. Se determinó la demanda química de oxígeno DQO usando un termoreactor de marca Velp Eco8 para $150 \pm 2 \stackrel{\circ}{C}$ y un espectrofotómetro marca Metash UV-Visible para leer a $610 \mathrm{~nm}$. 


\subsection{Adaptación del inóculo}

La muestra de ARLA, luego de ser recolectarla, se guardó por 1 semana en la cámara termostática a $35^{\circ} \mathrm{C}$, para adaptarla a la temperatura del ensayo (Nakasima-López et al., 2017). Se verificó la actividad del inóculo diariamente mediante su desgasificación.

\subsection{Ensayo de potencial de biogas por el método de jeringa}

El método empleado para la determinación del biogas es el de Hohenheim denominado HBT (DIN, 2006), el cual consiste en utilizar jeringas como biodigestores; a las que se le agrega el sustrato y el inóculo. El ensayo se mantiene a temperatura constante y se registra el volumen generado de biogas en un tiempo determinado. La cámara para termostatizar fue construida en el laboratorio de Bioprocesos de la Universidad Técnológica Nacional Facultad Regional Neuquén. Se usó un sistema de control Arduino y un caloventor 2000 watt, el cual permite trabajar a una temperatura constante de $35^{\circ} \mathrm{C}$.

Para la caracterización se emplearon 3 réplicas con la misma concentración de residuos (Hidalgo et al., 2014). Se usaron jeringas plásticas de $60 \mathrm{ml}$ descartables. Las concentraciones ensayadas fueron: $5 \%, 10 \%, 15 \%, 20 \%, 40 \%$ ST de FORSU y un blanco de inóculo solo. La producción de biogas se comprueba mediante combustión por llama directa.

\subsection{Inóculo para digestato y fitotoxicidad}

El reciclaje de los residuos mediante el proceso de compostaje convierte los residuos en un producto favorecedor para la recuperación y fertilización del suelo en la agricultura. El digestato que se produce en la digestion anarobia debe pretratarse por compostaje para aportar microorganismos y nutrientes al suelo de forma óptima (Medina Lara et al., 2017). El pretratamientamiento del compostaje se efectua con inóculo aerobio preparado a partir de residuos orgánicos. Para preparar el mismo se construyeron, compostadores de $30 \mathrm{l}$. El procedimiento consistió en agregar restos de poda (RP) como hojas secas y cesped, en capas alternadas de 5 a $10 \mathrm{~cm}$ apróximadamente mezclados con FORSU, y compost de 67 días. El acelerante permitió que el tiempo de compostaje fuese de 30 días. Utilizando el test de Zucconi por germinación de semillas de Lactuca sativa "lechuga" se evalúo la madurez del compost y la fitotoxicidad. Este índice de germinación IG indica el rango de toxicidad si: IG $\leq 50 \%$ toxicidad severa, $50 \%<\mathrm{IG}<80 \%$ toxicidad moderada, $50 \%<\mathrm{IG}<80 \%$ ausencia de toxicidad y IG $>100 \%$ estimulante, por lo cual el objetivo es observar si se obtienen valores mayores a $100 \%$.

\section{Resultados}

Los valores de la composición de residuos varían según la población, sus costumbres y poder adquisitivo. Se observa en la Tabla 1 que a fracción orgánica corresponde a $44.2 \%$ un porcentaje mayor que el resto. En general, se aprecian valores altos de FORSU en países subdesarrollados. Además, en Latinoamérica según Saez (2014) "el porcentaje de material putrescible es elevado", según datos publicados la fracción orgánica de los residuos oscila entre un 44 \% y 75 $\%$. A su vez, las ciudades argentinas que tienen entre 200 y 500 mil habitantes cuenta con un promedio de $34.91 \%$ de FORSU (Deluca et. al, 2015) , por lo cual, la cantidad de FORSU en la ciudad del Neuquén (NQN) es un 5\% más alta.

\begin{tabular}{|c|c|}
\hline $\mathbf{N}=\mathbf{6 0}$ & FORSU-NQN \\
\hline Papel & 5.2 \\
\hline Cartón & 6.0 \\
\hline Plástico & 10.9 \\
\hline FORSU & 44.2 \\
\hline Ferrosos & 0.5 \\
\hline Aluminio & 0.6 \\
\hline Vidrio & 4.6 \\
\hline
\end{tabular}

Tabla 1: Caracterización de residuos ciudad Neuquén ASTM D5231-92 (2016)

Los valores de caracterización de sustrato FORSU-NQN y del agua residual empleada se muestran en la Tabla 2. 


\begin{tabular}{|c|c|c|}
\hline $\mathbf{N = 3}$ & FORSU-NQN & AR LA \\
\hline $\mathrm{pH}\left(20^{\circ} \mathrm{C}\right)$ & 5.16 & 7.39 \\
\hline Conductividad $\left(21^{\circ} \mathrm{C}\right)$ & $2 \mathrm{~ms} / \mathrm{cm}^{2}$ & $1002 \mathrm{us} / \mathrm{cm}^{2}$ \\
\hline Sólidos Totales $(\mathrm{ST})$ & $67.12 \%$ & $0.687 \mathrm{mg} / \mathrm{l}$ \\
\hline Sólidos volátiles & $17.37 \%$ & $0.171 \mathrm{mg} / \mathrm{l}$ \\
\hline $\mathrm{DQO}$ & $13017 \pm 152 \mathrm{mg} / \mathrm{l} \mathrm{O}_{2}$ & $6839+-213 \mathrm{mg} / \mathrm{I} \mathrm{O}_{2}$ \\
\hline Acidez & $783 \pm 29 \mathrm{mg} / \mathrm{l} \mathrm{CaCO} 3$ & $93 \pm 29 \mathrm{mg} / \mathrm{l}$ \\
\hline
\end{tabular}

Tabla 2: Caracterización de FORSU-NQN y agua residual ARLA

Al realizar el ensayo de potencial de biogas, se determinó una mayor producción de metano para las concentraciones de $5 \%$ y $20 \%$ de FORSU con inóculo ARLA como se aprecia en la Fig. 1. Si aumenta la cantidad de sólidos totales entonces aumenta la cantidad de biogas generado (Yi et al., 2014) y, por otro lado, si la relación sustrato e inóculo S/I es menor a 1 hay también mayor producción de biogas (Cárdenas-Cleves et al., 2016). La única concentración con relación $\mathrm{S} / \mathrm{I}<1$ es la de $5 \%$ FORSU. Esta razón justifica que sean aproximadamente iguales para un $5 \%$ y $20 \%$ de ST FORSU. Además, se puede ver en dicha figura que el biogas generado es mayor a medida que crece el porcentaje de ST desde $10 \%$ al $20 \%$. En el caso del $40 \%$ de FORSU no se produjo biogas, y se debe a alguna sustancia inhibidora. Dado que cuando se aumenta la carga orgánica, se presentan efectos que evitan la generación de metano.(Pabón Pereira et al., 2012).

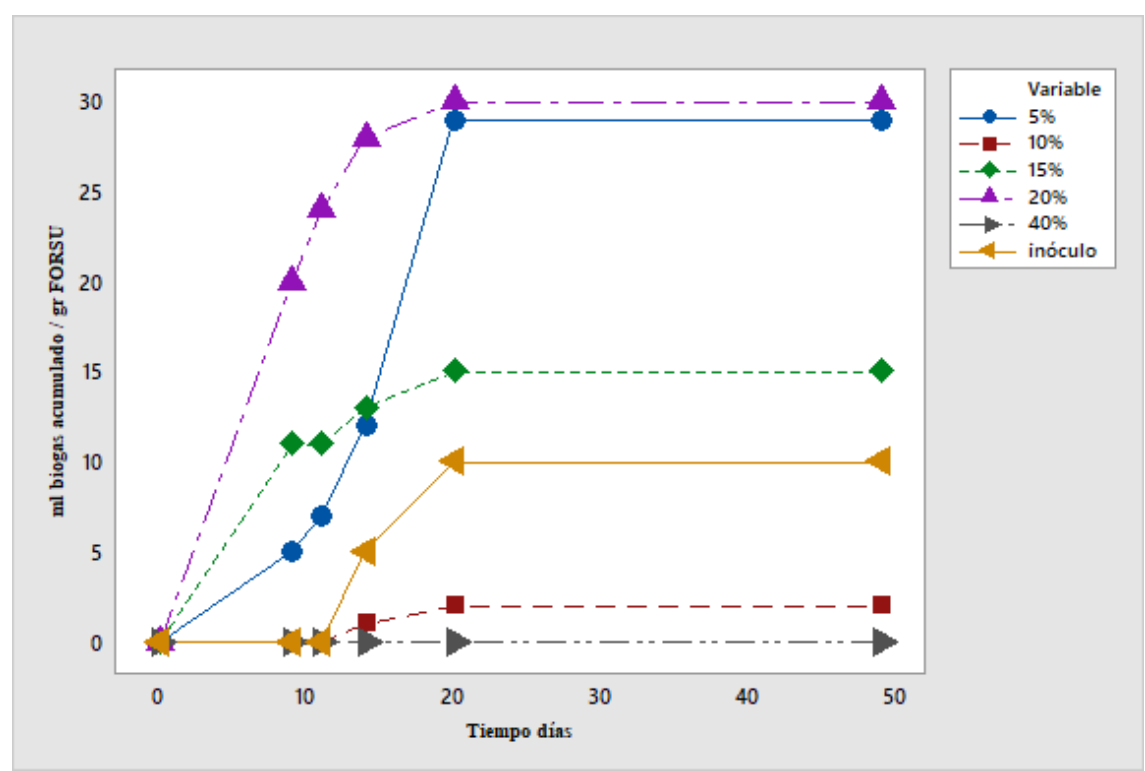

Fig. 1: Cantidad de biogas ml por día.

Para la preparación de inoculante de digestato se demoró 30 días y se obtuvieron los siguientes resultados registrados en la Tabla 3. Luego de realizar el ensayo fitoxicidad se observa que todos los compost evaluados tienen un valor mayor a $100 \%$ IG.

\begin{tabular}{|c|c|c|c|c|}
\hline & Agua & Compost 1 & Compost 2 & Compost 3 \\
\hline $\mathrm{pH}$ & 7.44 & 8.59 & 8.43 & 7.08 \\
\hline Conductividad eléctrica (CE) $\mu \mathrm{s} / \mathrm{cm}^{2}$ & 391 & 4530 & 4180 & 3280 \\
\hline Promedio de semillas germinadas & 4 & 4,7 & 6 & 7 \\
\hline Promedio de elongación raíz & 1.7 & 3.1 & 2.4 & 2.2 \\
\hline \% De germinación de semillas & 100 & 117,5 & 150 & 175 \\
\hline \% De elongación de las raíces (CRR) & 100 & 182,35 & 141,17 & 129,41 \\
\hline \%Índice de germinación (IG) & 100 & 214,26 & 211,75 & 226,46 \\
\hline
\end{tabular}

Tabla 3: Índice de germinación 


\section{Conclusiones}

Neuquén produce una fracción orgánica de residuos del 44,2 \% del total de los residuos municipales.

La muestra FORSU tiene $\mathrm{pH}=5,18 \pm 0$, una conductividad: $2 \mathrm{~ms} / \mathrm{cm}^{2}, \mathrm{ST}=67.12 \%, \mathrm{SV}=17.37 \%, \mathrm{DQO}=13017 \pm$ $152 \mathrm{mgO}_{2} / \mathrm{l}$, acidez total $=783 \pm 29 \mathrm{mg} / \mathrm{l} \mathrm{CaCO3}, \mathrm{NTK}=11 \%$. El inóculo empleado en $\mathrm{n}=3$ ARLA tiene $\mathrm{pH}=6 \pm 0$, una conductividad $=1002 \mathrm{~ms} / \mathrm{cm}^{2} . \mathrm{ST}=0.687 \mathrm{mg} / \mathrm{l}, \mathrm{SV}=0.171 \mathrm{mg} / \mathrm{l}$, acidez total $=93 \pm 29 \mathrm{mg} / \mathrm{l} \mathrm{CaCO}_{3}$. La cantidad de biogas acumulada es similar para concentraciones de $5 \%$ y $20 \%$ ST FORSU. La cantidad de biogas generado para 10 $\%, 15 \%$ y 20 \% ST FORSU aumenta con el aumento de los sólidos totales. No se evidenció producción de biogas en el ensayo con $40 \%$ de sólidos por inhibición.

El compost con acelerante es óptimo para su uso en 30 días. Todos los compost analizados tuvieron IG mayor a 100, por lo tanto, se determina que son estimulantes para la germinación de semillas y no tienen fitotoxicidad.

\section{Referencias}

APHA. 1989. Standard methods for the examination of water and wastewater. American Public Health Association. Washington DC.

Azim, K., Soudi, B., Boukhari, S., Perissol, C., Roussos, S., \& Thami Alami, I. (2018). Composting parameters and compost quality: a literature review. Organic Agriculture, 8(2), 141-158. https://doi.org/10.1007/s13165-017-0180-z

Barragan, H., Pascual, A., Bourgeois, M. J., \& Ojea, O. A. (2010). Desarrollo, salud humana y amenazas ambientales. http://sedici.unlp.edu.ar/handle/10915/26595

Cárdenas Cleves, L. M., Parra Orobio, B. A., Torres Lozada, P., \& Vásquez Franco, C. H. (2016). Perspectivas del ensayo de Potencial Bioquímico de Metano - PBM para el control del proceso de digestión anaerobia de residuos. Revista ION, 29(1), 95-108. https://doi.org/10.18273/revion.v29n1-2016008

Carneiro, T. F. (2005). Digestión anaerobia termofílica seca de residuos sólidos urbanos:

Deluca, M., \& Giorgi, N. (2015). Estudio de estrategia y factibilidad de residuos sólidos urbanos (rsu) para la republica Argentina.

DIN. (2006).VDI_4630 Fermentation of organic materials. Characterisation of the substrate, sampling, collection of material data, fermentation tests.

Gallardo-Izquierdo, Antonio; Laines-Canepa, José Ramón; Colomer-Mendoza, Francisco J; Miralles-Montins, Tamara; GómezParra, A. (2013). Biometanización de los rechazos procedentes del proceso de afino del compost. V Simposio

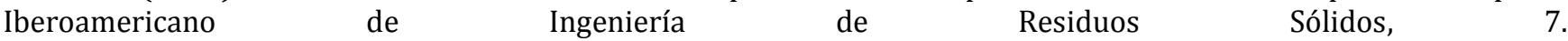
http://www.redisa.net/doc/artSim2013/TratamientoYValorizacionDeResiduos/Biometanizacion_Rechazos Procedentes Afino Compost.pdf

Holliger, C., Alves, M., Andrade, D., Angelidaki, I., Astals, S., Baier, U., Bougrier, C., Buffière, P., Carballa, M., De Wilde, V., Ebertseder, F., Fernández, B., Ficara, E., Fotidis, I., Frigon, J. C., De Laclos, H. F., Ghasimi, D. S. M., Hack, G., Hartel, M., ... Wierinck, I. (2016). Towards a standardization of biomethane potential tests. Water Science and Technology, 74(11), 2515-2522. https://doi.org/10.2166/wst.2016.336

MDH. (1996). Normas de Residuos Sólidos NB 742-760. La Paz: Ministerio de Desarrollo Humano. Secretaría de Participación Popular. Subsecretaría de Desarrollo Urbano. Dirección Nacional de Saneamiento Básico (MDH).

Nakasima-López, M., Taboada-González, P., Aguilar-Virgen, Q., \& Velázquez-Limón, N. (2017). Adaptación de Inóculos Durante el Arranque de la Digestión Anaerobia con Residuos Sólidos Orgánicos. Informacion Tecnologica, 28(1), 199208. https://doi.org/10.4067/S0718-07642017000100020

Neuquén, P. del. (2015). Estadística y Censos - Neuquén. https://www.estadisticaneuquen.gob.ar/\#/proyecciones_app

Pabón Pereira, C. P., Castañares, G., \& Van Lier, J. B. (2012). An OxiTop ${ }^{\circledR}$ protocol for screening plant material for its biochemical methane potential (BMP). Water Science and Technology, 66(7), 1416-1423. https://doi.org/10.2166/wst.2012.305

Pramanik, S. K., Suja, F. B., Zain, S. M., \& Pramanik, B. K. (2019). The anaerobic digestion process of biogas production from food waste: Prospects and constraints. Bioresource Technology Reports, 8(July), 100310. https://doi.org/10.1016/j.biteb.2019.100310

Rattanapan, C., Sinchai, L., Suksaroj, T. T., Kantachote, D., \& Ounsaneha, W. (2019). Biogas production by co-digestion of canteen food waste and domestic wastewater under organic loading rate and temperature optimization. Environments - MDPI, 6(2), 16. https://doi.org/10.3390/environments6020016

Saez, A. y U. G. (2014). Manejo de residuos sólidos en América Latina y el Caribe. Omnia, 20, 121-135. https://www.redalyc.org/pdf/737/73737091009.pdf

Toledo, R., Energética, F. P.-I., \& 2019, undefined. (2019). Potencial Energético de Codigerir Estiércol bovino, Lodos cloacales y residuos de Comida/Energy Potential to Codigest Cattle manure, sewage sludge and. Rie.Cujae.Edu.Cu, 40, 20-29. http://rie.cujae.edu.cu/index.php/RIE/article/download/550/615

Van, D. P., Fujiwara, T., Tho, B. L., Toan, P. P. S., \& Minh, G. H. (2020). A review of anaerobic digestion systems for biodegradable waste: Configurations, operating parameters, and current trends. Environmental Engineering Research, 25(1), 1-17. https://doi.org/10.4491/eer.2018.334 
Villalba, L., Santiago, R., Eloy, N., \& Basualdo, C. (2020). Resources , Conservation \& Recycling Household solid waste characterization in Tandil ( Argentina ): Socioeconomic, institutional , temporal and cultural aspects in fl uencing waste quantity and composition. Resources, Conservation \& Recycling, 152(July 2019 ), 104530. https://doi.org/10.1016/j.resconrec.2019.104530

Westerholm, M., Liu, T., \& Schnürer, A. (2020). Comparative study of industrial-scale high-solid biogas production from food waste: Process operation and microbiology. Bioresource Technology, 304. https://doi.org/10.1016/j.biortech.2020.122981

Yi, J., Dong, B., Jin, J., \& Dai, X. (2014). Effect of increasing total solids contents on anaerobic digestion of food waste under mesophilic conditions: Performance and microbial characteristics analysis. PLoS ONE, 9(7). https://doi.org/10.1371/journal.pone.0102548

Zucconi,F,Pera, A, Forte M, de Bartoldi, M, 1981. Evaluating toxicity in inmature compost. Biocicle 22:54-57. 